\title{
THE NONEXISTENCE OF EXPANSIVE HOMEOMORPHISMS OF 1-DIMENSIONAL COMPACT ANRS
}

\author{
HISAO KATO
}

(Communicated by James E. West)

\begin{abstract}
It is well known that if $X$ is an arc or a circle, then there is no expansive homeomorphism on $X$ (see [2] and [3]). In this note, we show that if $X$ is a Peano continuum which has a neighborhood $M$ such that $\operatorname{cl}(M)$ is a 1-dimensional AR, then there is no expansive homeomorphism on $X$. In particular, no 1-dimensional compact ANR admits an expansive homeomorphism.
\end{abstract}

\section{INTRODUCTION}

Let $X$ be a compact metric space with metric $d$. A homeomorphism $f$ of $X$ is expansive if there exists $c>0$ (called an expansivity constant for $f$ ) such that $d\left(f^{n}(x), f^{n}(y)\right) \leq c$ for all integer $n$ implies $x=y$. It is well known that the 2-adic solenoid and the 2-torus admit expansive homeomorphisms (see [6] and [7]). Also, Bryant [2] proved that there is no expansive homeomorphism on an arc. Jakobsen and Utz proved that there is no expansive homeomorphism on a circle [3].

By using those results, Kawamura showed that if $X$ is a Peano continuum and $X$ contains a free arc, then $X$ does not admit an expansive homeomorphism [4]. Naturally, the following problem arises: If $X$ is a 1-dimensional compact AR (or 1-dimensional compact ANR), is it true that $X$ does not admit an expansive homeomorphism? It is well known that there are may kinds of 1-dimensional compact ARs (or 1-dimensional compact ANRs) which contain no free arcs. In this note, we prove that if $X$ is a Peano continuum which has a neighborhood $M$ such that $\operatorname{cl}(M)$ is a 1-dimensional AR, then there exists no expansive homeomorphism on $X$. In particular, no 1-dimensional compact ANR admits an expansive homeomorphism. The proof is different from the proofs of Bryant, Jakobsen and Utz ([2] and [3]).

Received by the editors April 22, 1988 and, in revised form, February 20, 1989.

1980 Mathematics Subject Classification (1985 Revision). Primary 58F15; Secondary 54F50.

Key words and phrases. Expansive homeomorphism, dendrite (=1-dimensioinal compact AR), 1-dimensional compact ANR. 


\section{SELF-HOMEOMORPHISMS OF DENDRITES}

In this section, we prove that if $X$ is a dendrite (=1-dimensional compact AR), then there exists no expansive homeomorphism on $X$. To prove this result, we use an idea of Mañé [5].

2.1. Lemma [1,(13.5)]. A continuum $X$ is a 1-dimensional AR if and only if $X$ is a nondegenerate Peano continuum and $X$ contains no circle.

2.2. Lemma [5, Lemma 2.I]. Let $f: X \rightarrow X$ be an expansive homeomorphism of a compact metric space $X$ and let $c>0$ be an expansivity constant for $f$ and $0<\varepsilon<c / 2$. Then there exists $\delta>0$ such that if $x, y \in X, d(x, y)<$ $\delta$, and $\varepsilon \leq \sup \left\{d\left(f^{j}(x), f^{j}(y)\right) \mid 0 \leq j \leq n\right\} \leq 2 \varepsilon$ for some $n \geq 0$, then $d\left(f^{n}(x), f^{n}(y)\right) \geq \delta$.

2.3. Theorem. Let $X$ be a 1-dimensional compact AR. If $f: X \rightarrow X$ is a homeomorphism, then $f$ is not expansive.

Proof. Suppose, on the contrary, that $f$ is expansive. Let $\delta>0$ be as in 2.2. Since $X$ is locally connected, there are subcontinua $X_{1}, X_{2}, \ldots, X_{m}$ of $X$ such that $X=\cup X_{i}$ and $\operatorname{diam} X_{i}<\delta$ for each $i$. Let $X_{i, j}^{n}=f^{n}\left(X_{i}\right) \cap f^{-n}\left(X_{j}\right)$. Since $f^{n}\left(X_{i}\right)$ and $f^{-n}\left(X_{j}\right)$ are subcontinua in $X$ and $X$ contains no circle, $X_{i, j}^{n}=f^{n}\left(X_{i}\right) \cap f^{-n}\left(X_{j}\right)$ is connected (it may be empty). By the proof of [5, p. 318-319], we can conclude that $\lim _{n \rightarrow \infty}\left(\sup _{i, j} \operatorname{diam} X_{i, j}^{n}\right)=0$. Note that $X=\cup\left\{X_{i, j}^{n} \mid 1 \leq i, j \leq m\right\}$. Since $\operatorname{diam} X \leq \sum_{i, j=1}^{m} \operatorname{diam} X_{i, j}^{n}$, we can conclude that $X$ is degenerate, which is a contradiction.

\section{Self-homeomorphisms of certain Peano continua}

In this section, we prove that if $X$ is a Peano continuum and $X$ contains a neighborhood $M$ such that $\operatorname{cl}(M)$ is a 1-dimensional AR, $X$ does not admit an expansive homeomorphism. In particular, no 1-dimensional compact ANR admits an expansive homeomorphism.

3.1. Lemma [1, (13.6)]. A continuum $X$ is a 1-dimensional ANR if and only if $X$ is a Peano continuum of dimension 1 and $X$ does not contain infinite circles.

3.2. Theorem. If $X$ is a Peano continuum which has a neighborhood $M$ such that $\mathrm{cl}(M)$ is a 1-dimensional $\mathrm{AR}$, then $X$ does not admit an expansive homeomorphism. In particular, no 1- dimensional compact ANR admits an expansive homeomorphism.

Proof. By 2.1, $\operatorname{cl}(M)$ is a dendrite. Choose nonempty open sets $U_{1}, U_{2}$ in $M$ such that $\mathrm{cl} U_{1} \subset U_{2} \subset \operatorname{cl} U_{2} \subset M$. Let $Y$ be a component of $\operatorname{cl} U_{1}$ such that $U_{1} \cap Y \neq \varnothing$. Note that $Y$ is a dendrite. Suppose, on the contrary, that there is an expansive homeomorphism $f: X \rightarrow X$. let $\delta>0$ be as in 2.2 and let $X_{1}, X_{2}, \ldots, X_{m}$ be subcontinua of $X$ such that $X=\bigcup_{i=1}^{m} X_{i}$ and $\operatorname{diam} X_{i}<\delta$ 
for each $i$. Note that there is a natural number $p>0$ such that if $Z$ is a subcontinuum of $X$, then the number of components of $Z \cap Y$ is less than $p$. Suppose, on the contrary, that there is a sequence $\left\{Z_{n}\right\}$ of subcontinua of $X$ such that $\mid\left\{C \mid C\right.$ is a component of $\left.Y \cap Z_{n}\right\} \mid \geq n$, where $|A|$ denotes the cardinal number of a set $A$. Since $M$ does not contain a circle, we can see that $\mid\left\{C \mid C\right.$ is a component of $\left(\operatorname{cl} U_{2}-U_{1}\right)$ with $\left.C \cap \mathrm{cl} U_{1} \neq \varnothing \neq C \cap X-U_{2}\right\} \mid=\infty$. This implies that $X$ is not locally connected, which is a contradiction.

Now, let $X_{i, j}^{n}=f^{n}\left(X_{i}\right) \cap f^{-n}\left(X_{j}\right) \cap Y$. Since $Y$ contains no circle, we can conclude that the number of components of $X_{i, j}^{n}$ is less than $p^{2}$. By the proof of [5, p. 318-319], $\lim _{n \rightarrow \infty} \operatorname{diam} C_{i, j}^{n, k}=0$, where $C_{i, j}^{n, k}$ is a component of $X_{i, j}^{n}$. Since $Y=\bigcup C_{i, j}^{n, k}$, we can conclude that $\operatorname{diam} Y=0$. This is a contradiction.

Question 1. Is it true that Menger's universal curve admits an expansive homeomorphism?

Question 2. If $X$ is a dendroid (= pathwise connected treelike continuum) or a treelike continuum, is it true that $X$ does not admit an expansive homeomorphism?

Added in proof. Recently, the author proved that there are no expansive homeomorphisms on hereditarily decomposable treelike (or circlelike) continua.

\section{REFERENCES}

1. K. Borsuk, Theory of retracts, Monografie Matematyczne 44, Polish Scientific Publishers, Warszawa, 1967.

2. B. F. Bryant, Unstable self-homeomorphisms of a compact space, Vanderbilt University Thesis, 1954.

3. J. F. Jakobsen and W. R. Utz, The nonexistence of expansive homeomorphisms of a closed 2-cell, Pacific J. Math. 10 (1960), 1319-1321.

4. K. Kawamura, $A$ direct proof that each Peano continuum with a free arc admits no expansive homeomorphisms, Tsukuba J. Math. 12 (1988), 521-524.

5. R. Mañé, Expansive homeomorphisms and topological dimension, Trans. Amer. Math. Soc. 252 (1979), 313-319.

6. R. F. Williams, A note on unstable homeomorphisms, Proc. Amer. Math. Soc. 6 (1955), 308309.

7. T. O'Brien and W. Reddy, Each compact orientable surface of positive genus admits an expansive homeomorphism, Pacific J. Math. 35 (1970), 737-741.

Faculty of Integrated ARts and Sciences, Hiroshima University, HigashisendaMachi, Naka-Ku, Hiroshima 730, Japan 